\title{
Analysis of performance in Depth Based Routing for Underwater Wireless Sensor Networks
}

\author{
Simonetta Balsamo ${ }^{1}$, Dieter Fiems ${ }^{2}$, Mohsin Jafri $^{1}$, and Andrea Marin ${ }^{1}$ \\ 1 DAIS, Università Ca' Foscari Venezia, Italy \\ \{balsamo,mohsin.jafri,marin\}@unive.it \\ 2 Departement of Telecommunications and Information Processing, \\ Ghent University, Belgium \\ Dieter.Fiems@UGent.be
}

\begin{abstract}
In the last decade, Underwater Wireless Sensor Networks (UWSNs) have been widely studied because of their peculiar aspects that distinguish them from common wireless terrestrial networks. In fact, most UWSNs use acoustic instead of radio-frequency based communications, and nodes are subject to high mobility caused by water currents. As a consequence, specialised routing algorithms have been developed to tackle this challenging scenario. Depth based Routing (DBR) is one of the first protocols that have been developed to this aim, and is still widely adopted in actual implementations of UWSNs. In this paper we propose a stochastic analysis that aims at evaluating the performance of UWSNs using DBR in terms of expected energy consumption and expected end-to-end delay. Under a set of assumptions, we give expressions for these performance indices that can be evaluated efficiently, and hence they can be adopted as the basis for optimizing the configuration parameters of the protocol.
\end{abstract}

\section{Introduction}

Underwater Wireless Sensor Networks (UWSNs) [2] are used for a large number of applications which range from environmental monitoring to military purposes. They share with their terrestrial counterparts some important factors including the high importance of energy preservation at the nodes. This need is due to the fact that nodes are autonomous and equipped with a battery that is difficult or expensive to replace. Some research efforts have been done in the direction of developing sensor nodes with the capability of harvesting energy from the environment, however the results seem to be still at their early stages $[19,10$, 9]. However, in contrast with terrestrial wireless sensor networks, most of the UWSNs adopt acoustic communication instead of the traditional one based on radio-frequency. This implementation choice is due to the fact that acoustic communications cover long distances with low energy and are less prone to the problem of interferences. However, it also poses some severe limitations on the available bandwidth and on the propagation delay that is much higher than that measured in terrestrial electromagnetic radio-frequency based networks. As 
a consequence, most of the methods developed for the performance evaluation of terrestrial networks (see, e.g., $[3,15,6]$ ) cannot be straightforwardly applied to underwater networks. Another characteristic of UWSN is that nodes are subject to high mobility caused by water currents. As a consequence, routing schemes that adopt the flooding strategy only in the routing discovery phase and then store the sequence of nodes to the destination in memory (e.g., [12]), are not applicable. In the underwater scenario, routes continuously change and in most of the cases the best option is that of performing a controlled flooding for each packet transmission [7]. In order to tackle this problem, one of the earliest protocol that has been introduced is called Depth Based Routing (DBR) [21]. While we leave the detailed descriptions of its functionalities to Section 2, here we just recall few relevant aspects. DBR assumes that node does not know their position but are aware of their depth thanks to the presence of sensors of pressure. The goal of DBR is that of delivering a packet harvested in any part of the network to the sinks which float at the surface of the water. To this aim, the protocol relies on some algorithms among which one is inherited from the vehicular network design, i.e., one that is based on holding time. Basically, every node that receives a packet to be forwarded, waits a time which is inversely proportional to the depth difference from the source node.

There are several parameters that need to be set to configure a network employing DBR among which the major role is played by the transmission power. In the view of preserving the energy at the nodes, short transmissions seem to be more convenient, however we must take into account the fact that multiple forwarding will be required. Transmission power of a node depends on the distance required to cover through a single transmission [11]. On the other hand, long distance transmissions tend to drain the battery quickly. From the point of view of the reduction of the end-to-end delay, long transmissions are clearly optimal, but this requirement must always be balanced with that of a proper policy for energy saving.

In this paper we propose a model to study the impact of the transmission power on the total energy consumption of the network and on the end-to-end delay. The model is based on a set of results from the stochastic geometry research field [4] as well as on the manipulation of hypergeometric functions. We give detailed expressions for the expected energy cost and time cost per unit of distance of a certain transmission power. These expressions are extremely fast to evaluate and hence are appropriate for studying the optimal transmission power under certain network conditions.

The paper is structured as follows. In Section 2 we briefly introduce the main features of DBR. Section 3 presents our stochastic model and the derivation of the performance indices. In Section 4 we show some numerical results and illustrate an example of optimisation. In Section 5 we discuss some related work, and then Section 6 gives some final remarks. 


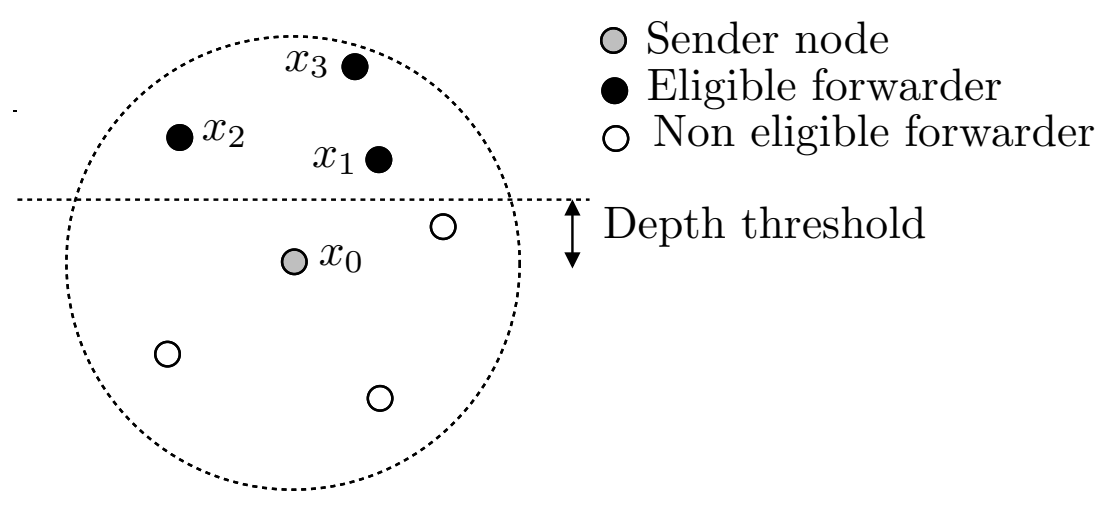

Fig. 1: Sketch of a transmission in DBR.

\section{Depth Based Routing}

DBR is an opportunistic routing protocol defined for UWSNs which works in a very simple manner. We give the explanation in the case of omnidirectional antennas following the schema of Figure 1. Recall that the goal of the model is that of delivering a packet to the nodes floating on the surface. Suppose that node $x_{0}$ transmits a packet that cannot reach the surface due to the limited transmission radius. The packet contains the depth of $x_{0}$ and each node that receives it correctly computes the depth difference between itself and $x_{0}$. One of the configuration parameters of the protocol is the depth threshold: every node whose depth difference is lower than the depth threshold is not an eligible forwarder. This parameter is used to prevent short range communications, but in some cases it can also assume negative values in order to avoid the problems connected with local minima in the network topology [14], i.e., situations in which a node wants to transmit a packet to the surface and is at a lowest depth than its neighbours but cannot reach the surface in one step. In our paper we assume the depth threshold to be 0, i.e., we assume all the nodes that have lower depth than the sender to be eligible forwarders. Among the eligible forwarders we choose the forwarding node as follows: each node computes a delay whose duration is inversely proportional to the depth difference. This delay is called holding time. Then, the node behaves as follows: it keeps the packet in its holding queue for the duration of the holding time. If during this period it listens to a retransmission of the packet, then it cancels it from its holding queue otherwise, at its expiration, it forwards the packet. In an ideal situation this mechanism has two effects: it prevents packet collisions and redundant transmissions and chooses the node that is closest to the surface as packet forwarder in a completely distributed way. In our paper we assume the holding time mechanism to work in the ideal way, in other words a node with depth difference $d$ will be a forwarder if and only if two conditions are satisfied:

1. It correctly receives the packet, 


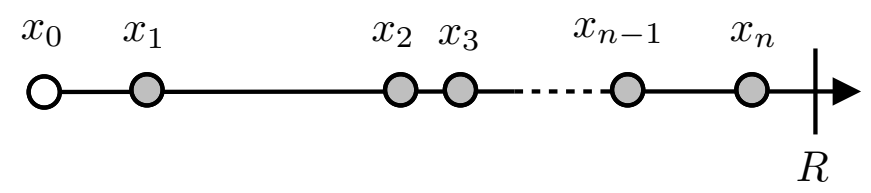

Fig. 2: Graphical representation of the model studied in Section 3.

2. All the nodes that are closer to the surface than itself fail to correctly receive the packet.

In the example of Figure 1, node $x_{1}$ will be the forwarder if and only if it correctly receives the packet sent by $x_{0}$ while $x_{2}$ and $x_{3}$ fail.

\section{A model for multi-hop protocol with directional antenna}

We consider a model where a node $x_{0}$ sends a data packet in a certain direction (e.g., toward the surface). Following convention of DBR[21], We assume that the locations of the candidate relay nodes on that direction are independent and uniformly distributed (see Figure 2). If we assume that we have $n$ potential relay nodes in a radius $R$, we can use the results from order statistics to characterise the distribution of the $i$-th node. Let $X_{(i)}^{*}$ be the random variable denoting the distance from $x_{0}$ to the $i$-th node, with $1 \leq i \leq n$. Then, the p.d.f. of $X_{(i)}^{*}$ is that of the marginal $i$-th order statistics of $n$ independent uniform random variables in the real interval $(0, R)$ that corresponds to a rescaled Beta distribution:

$$
f_{X_{(i)}}^{*}(x)=n\left(\begin{array}{c}
n-1 \\
i-1
\end{array}\right) \frac{x^{i-1}(R-x)^{n-i}}{R^{n}},
$$

where $x$ is the distance from $x_{0}$. For practical purposes, we cannot characterise the exact number of nodes $n$ in a distance $R$, but in many cases we know the expected density of the nodes. Hence, we let $n \rightarrow \infty$ and $R \rightarrow \infty$, as follows:

$$
\lim _{\substack{n \rightarrow \infty \\ R \rightarrow \infty}} \frac{n}{R}=\delta,
$$

where $\delta \in \mathbb{R}^{+}$is the expected number of nodes per unit of distance (density) of the network. Now, let $X_{(i)}$ be the r.v. associated with the distance between $x_{0}$ and the $i$-th node, and let $f_{X(i)}$ be its p.d.f.

Proposition 1. The p.d.f. of the location of the $i$-th node on the line in a network with density $\delta$ is given by:

$$
f_{X_{(i)}}(x)=\frac{\delta^{i} e^{-\delta x} x^{i-1}}{(i-1) !} .
$$


Proof. We have to compute the limit:

$$
\lim _{\substack{n \rightarrow \infty \\ R \rightarrow \infty}} f_{X_{(i)}}^{*}(x),
$$

under the constraint (1). We can write:

$$
\begin{gathered}
\lim _{\substack{n \rightarrow \infty \\
R \rightarrow \infty}} f_{X_{(i)}}^{*}(x)=\lim _{n \rightarrow \infty} n\left(\begin{array}{c}
n-1 \\
i-1
\end{array}\right) \frac{x^{i-1}\left(\frac{n}{\delta}-x\right)^{n-i}}{\left(\frac{n}{\delta}\right)^{n}}=\lim _{n \rightarrow \infty} \frac{n !}{(i-1) !(n-i) !} \\
\cdot \frac{x^{i-1}\left(\frac{n}{\delta}-x\right)^{n-i}}{\left(\frac{n}{\delta}\right)^{n}}=\frac{x^{i-1}}{(i-1) !} \lim _{n \rightarrow \infty} \frac{n !}{(n-i) !} \frac{\delta^{i}}{(n-\delta x)^{i}}\left(1-\frac{\delta x}{n}\right)^{n} \\
=\frac{\delta^{i} x^{i-1}}{(i-1) !} \underbrace{\lim _{n \rightarrow \infty} \frac{n !}{(n-i) !(n-\delta x)^{i}}}_{1} \underbrace{\lim _{n \rightarrow \infty}\left(1-\frac{\delta x}{n}\right)^{n}}_{e^{-\delta x}}=\frac{\delta^{i} e^{-\delta x} x^{i-1}}{(i-1) !},
\end{gathered}
$$

that completes the proof.

Notice that, according to Proposition 1 the p.d.f. of $X_{(i)}$ is that of a Gamma r.v. with shape $i$ and rate $\delta$. In other words, not surprisingly, we obtain a homogeneous Poisson point process on the line, where the distribution of the $i$-th distant node from the origin $x_{0}$ is given by the sum of $i$ independent exponential r.v.s with rate $\delta$.

The following proposition will play an important role in the analysis that we propose, since it gives the probability that all the nodes more distant than a certain threshold $y$ fail to receive a packet sent by $x_{0}$.

Proposition 2. The probability that all the nodes located after a certain threshold y fail to receive a packet sent by $x_{0}$ is:

$$
p f(y)=\exp \left(-\frac{\delta}{\lambda} e^{-\lambda y}\right) .
$$

Proof. Let us consider an arbitrary node whose location is conditioned in the interval $(y, R), y>0, R \rightarrow \infty$, and recall that we are assuming that a node at distance $x$ will successfully receive a packet sent by $x_{0}$ with probability $e^{-\lambda x}$, $\lambda>0$ [14]. Then we have that the probability that such a node fails to receive a packet is:

$$
p f s(y)=\int_{y}^{R}\left(1-e^{-\lambda x}\right) \frac{1}{R-y} d x=1+\frac{e^{-\lambda R}-e^{-\lambda y}}{\lambda(R-y)} .
$$

Therefore, we can compute the probability that all the nodes are farther than $y$ from $x_{0}$ fail to receive a packet (recall that both the location and the events of correct packet reception are independent) and let $R \rightarrow \infty$ :

$$
p f(y)=\lim _{\substack{n \rightarrow \infty \\ R \rightarrow \infty}}\left(1+\frac{e^{-\lambda R}-e^{-\lambda y}}{\lambda(R-y)}\right)^{n},
$$


that under the constraint (1) can be written as:

$$
p f(y)=\lim _{n \rightarrow \infty}\left(1+\frac{e^{-\lambda \frac{n}{\delta}}-e^{-\lambda y}}{\lambda\left(\frac{n}{\delta}-y\right)}\right)^{n},
$$

and rewritten as:

$$
\lim _{n \rightarrow \infty}\left(1+\frac{e^{-\frac{\lambda}{\delta} n} \delta}{\lambda n-\lambda y \delta}-\frac{1}{n} e^{-\lambda y} \frac{\delta}{\lambda-\frac{\lambda y \delta}{n}}\right)^{n} .
$$

Now the proof the proposition follows easily.

Example 1. Let us consider a network in which the maximum distance covered by a successfully received packet is $250 \mathrm{~m}$, i.e., $\lambda=1 / 250$ in a network with a density of 10 nodes per $\mathrm{km}$, i.e., $\delta=0.01$ nodes per meter. In Figure 3a we show the probability that all the nodes after a certain $y$ fail to receive a packet sent by $x_{0}, p f(y)$.

Notice that by setting $y=0$ in Equation (3) we can compute the probability that all the nodes fail to receive the packet as:

$$
p f(0)=e^{-\delta / \lambda} \text {. }
$$

Now recall that $i$-th node will be the forwarder of a packet if and only if the following conditions are satisfied:

1. Node $i$, placed at $X_{(i)}$, correctly receives the packet from $x_{0}$;

2. All the nodes $X_{(i+1)}, X_{(i+2)}, \ldots$ fail to receive the packet.

Hence, the probability $P_{i}$ that the forwarder will be the $i$-th node is:

$$
P_{i}=\int_{0}^{\infty} f_{X_{(i)}}(x) e^{-\lambda x} p f(x) d x,
$$

which can be obtained by the independence assumption of the event of correct reception of a packet. Since $E\left[X_{(i)}\right]=i / \delta$, we have that the expected distance covered by a transmission is:

$$
\bar{L}=\frac{\sum_{i=0}^{\infty} i P_{i}}{\delta}
$$

Unfortunately, it is not easy to find a closed form expression for $P_{i}$, but since we are interested just in an expectation we can proceed as follows. Let $T$ be the r.v. that takes value $i$ with probability $P_{i}$, then we have:

$$
\bar{L}=\frac{E[T]}{\delta} .
$$

The following proposition gives the expression for $E[T]$ and hence for $\bar{L}$. 
Theorem 1. In a network with density $\delta$ and probability of correct reception $e^{-\lambda x}$, with $x$ the distance between source and destination is $E[T] / \delta$ where the expected forwarder in the network $E[T]$ can be computed as:

$$
E[T]=1-e^{-\delta / \lambda}+\frac{\delta}{\lambda}\left(\gamma+E_{1}\left(\frac{\delta}{\lambda}\right)+\log \left(\frac{\delta}{\lambda}\right)\right),
$$

$\gamma \simeq 0.577216$ is Euler's constant, and

$$
E_{1}(x)=\int_{x}^{\infty} \frac{1}{t} e^{-t} d x
$$

is the exponential integral function [16, Ch. 6.2].

Proof. We compute $E[T]$ as $\sum_{i=1}^{\infty} \operatorname{Pr}\{T \geq i\}$. For $i \geq 2$ by conditioning on the position $x$ of the $(i-1)$ th node the probability that at least one node in $(x, \infty)$ will retransmit the packet, i.e.:

$$
\operatorname{Pr}\{T \geq i\}=\int_{0}^{\infty} f_{X_{(i-1)}}(x)\left(1-e^{-\frac{\delta e^{-\lambda x}}{\lambda}}\right) d x, \quad i \geq 2
$$

while $\operatorname{Pr}\{T \geq 1\}=1-e^{-\delta / \lambda}$. Integral (6) can be shown to converge to $1-$ ${ }_{(i-1)} F_{(i-1)}(\mathbf{a} ; \mathbf{b} ; z)$ where

$$
\mathbf{a}=\left(\frac{\delta}{\lambda}, \ldots, \frac{\delta}{\lambda}\right)
$$

and

$$
\mathbf{b}=\left(1+\frac{\delta}{\lambda}, \ldots, 1+\frac{\delta}{\lambda}\right)
$$

and $c=-\delta / \lambda$.

Remark 1. The generalized hypergeometric function ${ }_{p} F_{q}(\mathbf{a}, \mathbf{b} ; z)$, where $\mathbf{a}=$ $\left(a_{1}, \ldots, a_{p}\right), \mathbf{b}=\left(b_{1}, \ldots, b_{q}\right)$, is defined as:

$$
{ }_{p} F_{q}(\mathbf{a}, \mathbf{b} ; z)=\sum_{n=0}^{\infty} \frac{\left(a_{1}\right) n \cdots\left(a_{p}\right)_{n}}{\left(b_{1}\right)_{n} \cdots\left(b_{q}\right)_{n}} \frac{z^{n}}{n !},
$$

where $\mathbf{a} \in \mathbb{R}^{p}, \mathbf{b} \in \mathbb{R}^{q}, z \in \mathbb{C}$ and $\left(a_{i}\right)_{0}=1,\left(a_{i}\right)_{n}=a(a+1) \cdots(a+n-1)$ is the Pochhammer's symbol. It is well-known that if $p<q+1$ then the series converges (absolutely) for any finite $z$ [16, Ch. 16]. In our case, we have $p=q=i-1$ and hence the convergence is proved.

Now, by using the definition of generalised hypergeometric series (see Equation (7)), we can rewrite Integral (6) as:

$$
\begin{aligned}
\operatorname{Pr}\{T \geq i\}=1-\sum_{k=0}^{\infty}\left[\frac{(\delta / \lambda)_{k}}{(1+\delta / \lambda)_{k}}\right]^{i-1} & \left(-\frac{\delta}{\lambda}\right)^{k} \frac{1}{k !} \\
& =1-\sum_{k=0}^{\infty}\left(\frac{\delta}{\delta+k \lambda}\right)^{i-1}\left(-\frac{\delta}{\lambda}\right)^{k} \frac{1}{k !} .
\end{aligned}
$$


Notice that the last expression for $i=1$ gives exactly $1-e^{-\delta / \lambda}$ and hence we can extend its validity for $i \geq 1$. By noting that $\operatorname{Pr}\{T=i\}=\operatorname{Pr}\{T \geq i\}-\operatorname{Pr}\{T \geq$ $i+1\}$, and since the series are absolutely convergent, we have:

$$
\operatorname{Pr}\{T=i\}=\sum_{k=0}^{\infty}\left(\frac{\delta}{\delta+k \lambda}\right)^{i-1}\left(-\frac{\delta}{\lambda}\right)^{k}\left(\frac{-k \lambda}{\delta+k \lambda}\right) \frac{1}{k !} .
$$

Let us now compute $E[T]$ :

$$
E[T]=\sum_{i=1}^{\infty} \operatorname{Pr}\{T \geq i\}=\sum_{i=1}^{\infty}\left(1-\sum_{k=0}^{\infty}\left(\frac{\delta}{\delta+k \lambda}\right)^{i-1}\left(-\frac{\delta}{\lambda}\right)^{k} \frac{1}{k !}\right)
$$

that can be rewritten as:

$$
\begin{aligned}
\sum_{i=1}^{\infty}\left(1-1-\sum_{k=1}^{\infty}\left(\frac{\delta}{\delta+k \lambda}\right)^{i-1}\left(-\frac{\delta}{\lambda}\right)^{k}\right. & \left.\frac{1}{k !}\right) \\
& =-\sum_{k=1}^{\infty}\left(-\frac{\delta}{\lambda}\right)^{k} \frac{1}{k !} \sum_{i=1}^{\infty}\left(\frac{\delta}{\delta+k \lambda}\right)^{i-1}
\end{aligned}
$$

Observe that $\delta /(\delta+k \lambda)<1$ by hypothesis and hence we can write:

$$
E[T]=-\sum_{k=1}^{\infty}\left(-\frac{\delta}{\lambda}\right)^{k} \frac{1}{k !}\left(\frac{\delta+\lambda k}{\lambda k}\right) .
$$

We can rewrite Series (8) as:

$$
E[T]=-\left(\sum_{k=1}^{\infty}\left(-\frac{\delta}{\lambda}\right)^{k} \frac{1}{k !}\right)-\left(\sum_{k=1}^{\infty}\left(-\frac{\delta}{\lambda}\right)^{k} \frac{1}{k ! k} \frac{\delta}{\lambda}\right) .
$$

From the observation that:

$$
\sum_{k=1}^{\infty}\left(-\frac{\delta}{\lambda}\right) \frac{1}{k !}=e^{-\delta / \lambda}-1
$$

and by [16, Eq. 6.6.2]:

$$
\sum_{k=1}^{\infty}\left(-\frac{\delta}{\lambda}\right)^{k} \frac{1}{k ! k} \frac{\delta}{\lambda}=\frac{\delta}{\lambda} \sum_{k=1}^{\infty}\left(-\frac{\delta}{\lambda}\right)^{k} \frac{1}{k ! k}=-\frac{\delta}{\lambda}\left(\gamma+\log \left(\frac{\delta}{\lambda}\right)+E_{1}\left(\frac{\delta}{\lambda}\right)\right),
$$

the theorem is proved. 


\subsection{Analysis of the energy consumption}

In the light of Theorem 1 we can draw some conclusions on the optimal transmission power for the sender node. Recall that the probability for a node to correctly receive a packet sent at distance $d$ is $e^{-\lambda d}$, i.e., high values of parameter $\lambda$ model a system in which the expected transmission length is short. We model the energy consumed for a transmission as function of $\lambda$ as follows:

$$
\operatorname{En}(\lambda)=G \frac{1}{\lambda^{\alpha}},
$$

where $G$ is a normalising constant, and $\alpha$ is a parameter that depends on some environmental factors like the depth of the node, the salinity and the spreading coefficient [20]. Indeed, in underwater networks, the transmission power is consumed by the spreading of the signal and by its attenuation. The latter factor depends on the frequency adopted by the network as stated by Thorp's formula [5], but it tends to be lower for lower frequencies. The spreading coefficient, is lower than that of terrestrial networks, i.e., its value is between 1 and 2 . In our case we can assume $\alpha>1$, and we compute the expected energy consumption for unit of distance as:

$$
\operatorname{Cons}(\lambda)=\frac{E n(\lambda)}{\bar{L}},
$$

where $\bar{L}$ is computed thanks to Theorem 1 . It can be shown that for $\alpha>1$ (which is the case for practical scenarios), we have that $\operatorname{Cons}(\lambda) \rightarrow 0$ as $\lambda \rightarrow \infty$, whereas $\operatorname{Cons}(\lambda) \rightarrow \infty$ as $\lambda \rightarrow 0$. This confirms the idea that, from the point of view of the energy consumption, it is more convenient to perform many multi hop short transmissions rather than long transmissions. However, as we will see in Section 3.2, the energy consumption is not the only requirement of an UWSNs, i.e., we have to consider also the problem of the end-to-end delay. Short transmissions require the packet to be sent multiple times for a correct reception and the multi-hop mechanism used by protocols such as DBR requires to wait for the holding time to expire at each forwarding/retransmission step. We will study the trade off between energy saving and low end to end delay in the following sections. In Figure $3 \mathrm{~b}$ and $3 \mathrm{c}$ we show the plots of $\operatorname{Cons}(\lambda) / G$ for some practical values of $\alpha=1.2$ and $\alpha=1.5$ [17]. Notice that in the first plot the function still tends to $\infty$ for $\lambda \rightarrow 0$ even if the scale has been chosen to show the maximum around 100 .

\subsection{Analysis of the end-to-end delay}

In this section we introduce a performance index that measures the speed at which a packet is forwarded in the network. To this aim, we measure the expected time required by a packet to cover a unit of distance. Notice that when none of the eligible forwarders correctly receives the packet sent by $x_{0}$ this has to be resent and the holding time must be newly waited. Let $\tau$ be the time required 
by the transmission of the packet. Then, the expected time to cover a unit of distance is given by:

$$
\operatorname{Time}(\lambda)=\frac{\operatorname{Tr}(\lambda) \tau}{E[L \mid \text { correct transmission }]},
$$

i.e., the expected number of transmissions $\operatorname{Tr}(\lambda)$ required to get a successful packet forwarding multiplied by the time required by each transmission and divided by the conditional expectation of the distance covered by one transmission. By the independent assumption, $\operatorname{Tr}(\lambda)$ is the expected value of a geometric random variable whose failure probability is given by Equation (4). Equation (4) can be used also for the computation of the conditional expectation and after simplifying Equation (10) we obtain:

$$
\operatorname{Time}(\lambda)=\frac{\tau}{\bar{L}} .
$$

In Figure 3d we show the plot of $\operatorname{Time}(\lambda) / \tau$ for $\alpha=1.5$. As expected, we have that:

$$
\lim _{\lambda \rightarrow \infty} \operatorname{Time}(\lambda)=\infty, \quad \lim _{\lambda \rightarrow 0} \operatorname{Time}(\lambda)=0 .
$$

\section{Numerical evaluation and optimisation}

In this section we study a simple instance of optimization problem. From the previous sections, we observed that from the point of view of the expected energy consumption it is more convenient to perform multiple transmissions with small range to deliver a packet, whereas in order to minimize the end-to-end delay we should maximise the transmission power. We can study this trade off by introducing a cost function defined as the linear combination of these two performance indices:

$$
\operatorname{Cost}(\lambda)=a \cdot \operatorname{Cons}(\lambda)+b \cdot \operatorname{Time}(\lambda),
$$

where $a, b \geq 0$ are some weight constants. Considering that the propagation speed of acoustic signals in the underwater environment is $v_{0}=1500 \mathrm{~m} / \mathrm{s}$, we take $2 s$ for the sum of the holding time and the sending time. Since the energy consumption strongly depends on the technical implementation of the acoustic modems, we assume as basic unit of measure for the energy, the energy $\epsilon$ required to have $1 / \lambda=100 \mathrm{~m}$, which implies $G=1000$. We take $b=1 \mathrm{~m} / \mathrm{s}$ and $a$ varying from $0.5 \mathrm{~m} / \epsilon$ to $1.5 \mathrm{~m} / \epsilon$. In Figure $3 \mathrm{e}$ we show the graphical solution to the optimisation problem. Unfortunately, the minimisation of the cost function does not admit an explicit solution, however simple numerical approaches can be adopted to solve the problem.

We now study the relation between the node density and the optimal transmission range. Let $\lambda^{*}$ be the solution of the optimisation problem:

$$
\lambda^{*}=\operatorname{argmin}_{\lambda}\{\operatorname{Cost}(\lambda)\} .
$$


In Figure 4a we show $\lambda^{*}$ as function of $\delta$. We notice that for very low node densities, the model suggests a strategy that prefers to repeat many short range transmission, i.e., it relies on redundant transmissions to deliver a packet. The optimal transmission power has a maximum around $\delta=0.006$ but then it decreases again since the nodes are so dense that the protocol can achieve good performance with low energy. The fact that $\lambda^{*}$ is not a monotonic function of the node densities does not mean that the protocol cannot take advantage when we move from $\delta=0.002$ to 0.05 as shown by Figures $4 \mathrm{~b}$ and $4 \mathrm{c}$. These two figures show the plots of the expected energy consumption and delay per meter associated with $\lambda^{*}$. We observe that, as expected, higher node densities give lower costs and hence better performance.

\section{Related work}

Underwater networks have drawn a lot of attention in the latest years. The peculiarity of these networks requires to adjust the opportunistic protocols defined for vehicular networks. However, while for vehicular (terrestrial) networks, several analytical models have been proposed (see, e.g., [1]), less results are available for underwater networks. In [8] the authors optimize some performance indices for a generic multi hop protocol in UWSNs with respect to the optimal number of forwarding. With respect to this work, we focus on a specific protocol and solve the optimization problem based on its parameter configurations. In fact, given the optimal number of hops, it is not clear how one should configure the protocol parameters to reach that condition. In [13] the authors propose a performance evaluation of a Aloha-like communication protocol for UWSNs in a time-slotted setting. [18] proposes a Markovian model for a single acoustic channel but there is no consideration for the multi-hop behaviour of DBR.

\section{Conclusion}

In this paper we have proposed a model for an opportunistic routing protocol for UWSNs, namely DBR. Despite the assumptions done to allow for an analytical tractability of the performance indices, the model gives an insight on the impact of the parameter settings on the behaviour of the protocol. Specifically, two important performance indices have been considered: the expected energy consumption and delay for unit of distance covered by one hop transmission. Based on these performance indices, or on a combination of the two, one can optimise the most important parameter which is the transmission power. Future works include the extension of the model in order to cope with the 3D characteristic of omnidirectional antennas and to allow non homogeneous deployment of the network nodes. In the latter case, the model could be used also for determining the optimal node deployment for a homogeneous energy consumption of the nodes, and hence for a longer lifetime of the network. 


\section{References}

1. A. Abedi, M. Ghaderi, and C. L. Williamson. Distributed routing for vehicular ad hoc networks: Throughput-delay tradeoff. In Proc. of MASCOTS, pages 47-56, 2010.

2. I. F. Akyildiz, D. Pompili, and T. Melodia. Underwater acoustic sensor networks: Research challenges. Ad Hoc Networks, 3(3):257-279, 2005.

3. E. Ancillotti, R. Bruno, and M. Conti. Design and performance evaluation of throughput-aware rate adaptation protocols for IEEE 802.11 wireless networks. Performance Evaluation, 66(12):811-825, 2009.

4. F. Baccelli and B. Blaszczyszyn. Stochastic Geometry and Wireless Networks. Volume I - Theory. Foundations and Trends in Networking Vol. 3. NoW Publisher, 2009.

5. L. Berkhovskikh and Y. Lysanov. Fundamentals of Ocean Acoustics. Springer, 1982.

6. M. Bugliesi, L. Gallina, S. Hamadou, A. Marin, and Rossi S. Behavioural equivalences and interference metrics for mobile ad-hoc networks. Performance Evaluation, 73:41-72, 2014.

7. Rodolfo WL Coutinho, Azzedine Boukerche, Luiz FM Vieira, and Antonio AF Loureiro. Design guidelines for opportunistic routing in underwater networks. IEEE Communications Magazine, 54(2):40-48, 2016.

8. F. A. de Souza, B. Sens Chang, and G. Brante. Optimizing the number of hops and retransmissions for energy efficient multi-hop underwater acoustic communications. IEEE Sensors, pages 3927-3938, 2016.

9. H. D. Erdem and V. C. Gungor. Lifetime analysis of energy harvesting underwater wireless sensor nodes. In 25th Signal Processing and Communications Applications Conference, SIU 2017, Antalya, Turkey, May 15-18, 2017, pages 1-4, 2017.

10. E. Gelenbe and A. Marin. Interconnected wireless sensors with energy harvesting. In Analytical and Stochastic Modelling Techniques and Applications - 22nd International Conference, ASMTA 2015, Albena, Bulgaria, May 26-29, 2015. Proceedings, pages 87-99, 2015.

11. Albert F Harris III and Michele Zorzi. Modeling the underwater acoustic channel in ns2. In Proceedings of the 2nd international conference on Performance evaluation methodologies and tools, pages 18-26. ICST, 2007.

12. R.H. Jhaveri and N.M Patel. Mobile ad-hoc networking with aodv: A review. International Journal of Next-Generation Computing, 6(3):165-191, 2015.

13. D. Marinakis, K. Wu, N. Ye, and S. Whitesides. Network optimization for lightweight stochastic scheduling in underwater sensor networks. IEEE Transactions on Wireless Communications, 11(8):2786-2795, 2012.

14. Y. Noh, U. Lee, S. Lee, P. Wang, L. F. M. Vieira, J.-H. Cui, M. Gerla, and K. Kim. Hydrocast: pressure routing for underwater sensor networks. IEEE Transactions on Vehicular Technology, 65(1):333-347, 2016.

15. J. Nzouonta, T. Ott, and C. Borcea. Impact of queuing discipline on packet delivery latency in ad hoc networks. Performance Evaluation, 66(12):667-684, 2009.

16. F. W. J. Olver, D. W. Lozier, R. F. Boisvert, and Clark C. W. NIST Handbook of Mathematical Functions. Cambridge University Press, 2010.

17. Nathan Parrish, Leonard Tracy, Sumit Roy, Payman Arabshahi, and Warren LJ Fox. System design considerations for undersea networks: Link and multiple access protocols. IEEE Journal on Selected Areas in Communications, 26(9), 2008. 
18. F. Pignieri, F. De Rango, F. Veltri, and S. Marano. Markovian approach to model underwater acoustic channel: Techniques comparison. In Military Communications Conference, 2008. MILCOM 2008. IEEE, pages 1-7. IEEE, 2008.

19. B. Sai Srujana, Neha, Princy Mathews, and V.P. Harigovindan. Multi-source energy harvesting system for underwater wireless sensor networks. Procedia Computer Science, 46(Supplement C):1041 - 1048, 2015. Proceedings of the International Conference on Information and Communication Technologies.

20. R. Urick. Principles of Underwater Sound. McGraw-Hill, 1983.

21. Hai Yan, Zhijie Jerry Shi, and Jun-Hong Cui. Dbr: depth-based routing for underwater sensor networks. In International conference on research in networking, pages 72-86. Springer, 2008. 


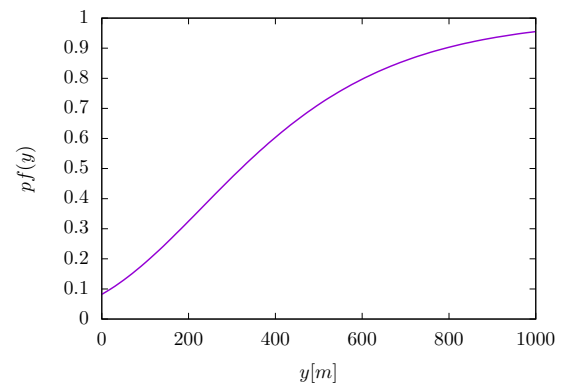

(a) Plot of the probability that none of the nodes more distant than $y$ meters from $x_{0}$ correctly receives a packet sent from $x_{0}$.

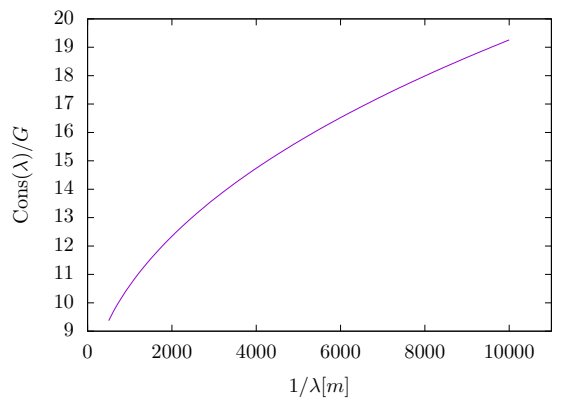

(c) Expected energy consumption per unit of distance for $\alpha=1.5$.

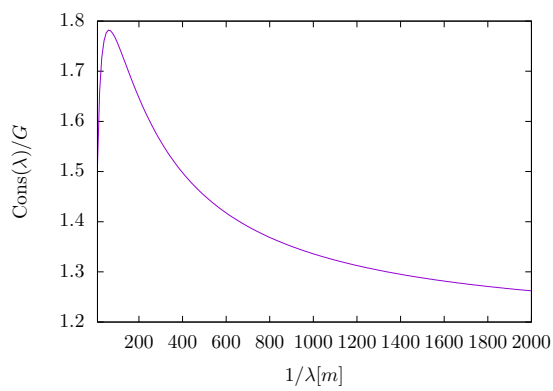

(b) Expected energy consumption per unit of distance for $\alpha=1.2$.

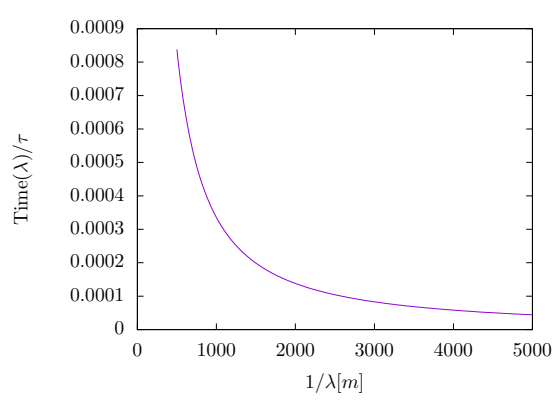

(d) Expected time per unit of distance for $\alpha=1.5$.

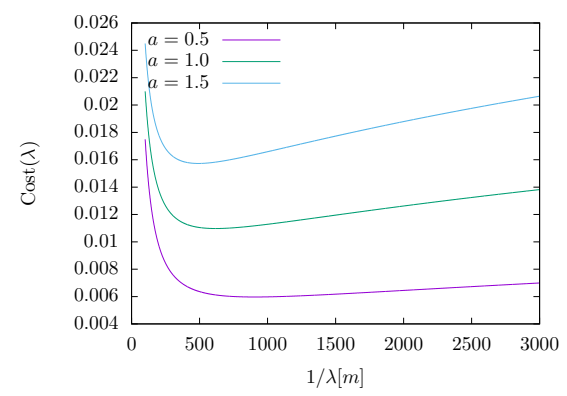

(e) Cost function for the example of Section 4.

Fig. 3: Table of plots. 


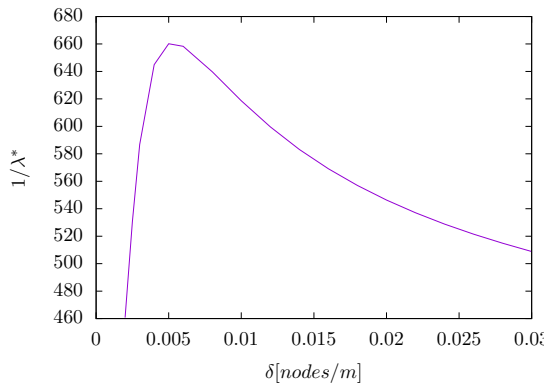

(a) Solution of the optimisation problem (11).

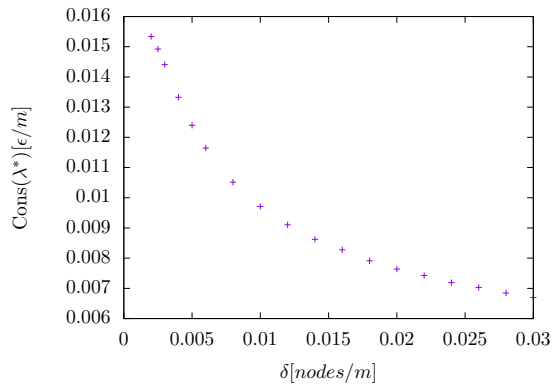

(b) Expected energy consumption per unit of distance for the optimal configuration.

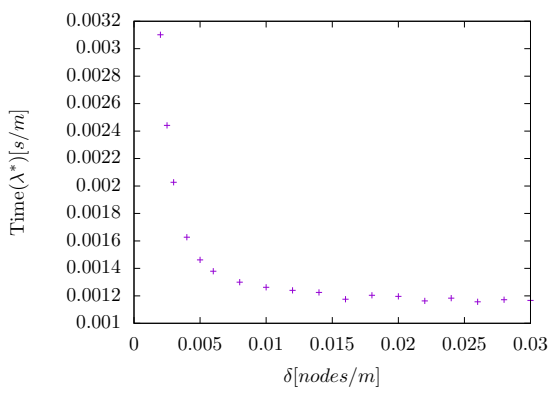

(c) Expected delay per unit of distance for the optimal configuration.

Fig. 4: Solution of the optimisation problem of Section 4. 